\title{
Caution should be taken when using electronic health record database
}

\author{
Lina Wang ${ }^{1}$ and Xiaoming $\mathrm{Wu}^{2^{*}}$ (D) \\ This comment refers to the article available at https://doi.org/10.1186/s13054-019-2465-y.
}

The weekend effect refers [1] to the difference in mortality rate between patients admitted to hospital on weekends and those admitted on weekdays. The weekend effect on patient outcomes has been a practical and research issue. ICU record has been extensively studied in terms of weekend mortality, but the results were not consistent and conclusive [2]. With this as background, Faust et al. [3] recently published an article about the weekend effect on ICU patients in Critical Care. The paper is of great interest, and we highly recognized the authors' logic in the data analysis of this paper.

However, we noted that the data used in the paper might be problematic, and consequently, the results may be unreliable. The cohort data of the paper were from the MIMIC-III database [4], and all admission dates in the database have been shifted to protect patients' privacy and comply with HIPAA (https://mimic.physionet.org/mimicdata/time/). Admission dates are only internally consistent for the same patient, and this deidentification method altered the real date. Therefore, it is not possible, after alternation, to determine whether a particular admission date was a weekend or not, unless the dates were drifted by adding indefinite integer number of weeks, but that was not mentioned in the description of the MIMIC-III database. One cannot judge whether a specific admission date is a weekend or not because of the drifted admission time in the database. For this reason, we believe that the cohort data in this paper may be inappropriate, and the subsequent results can be unreliable.

In conclusion, we suggest that when using MIMIC-III database for medical analysis, caution should be taken that the dates in the database have been drifted.

\section{Authors' response}

\section{Louis Faust, Keith Feldman and Nitesh V. Chawla}

We would like to first thank the authors for their concern regarding the reliability of the day of the week information in the MIMIC dataset.

While the authors are correct that the true admission dates in MIMIC are shifted, day of the week information has been retained and remains accurate. Unfortunately, note of this retention is absent from the official MIMIC database documentation that the authors (understandably) cite (https://mimic.physionet.org/mimicdata/time/). However, this information can be found on MIT's official Github, where the MIMIC data repository was utilized as part of the 2018 BHI \& BSN Data Challenge (https://github.com/MIT-LCP/bhi-bsn-challenge/blob/

\footnotetext{
* Correspondence: wxm@mail.xjtu.edu.cn

${ }^{2}$ Key Laboratory of Biomedical Information Engineering of Education Ministry, School of Life Science and Technology, Xi'an Jiaotong University, Xi'an

710049, Shaanxi, China

Full list of author information is available at the end of the article
}

master/challenge-demo.ipynb) specifically investigating the "weekend effect." As stated on the Github page, "A patient-level shift has been applied to dates. Day of week is retained."

Given the day of the week information is accurate, the results from the manuscript remain reliable.

\section{Abbreviations \\ HIPAA: Health Insurance Portability and Accountability Act; ICU: Intensive care unit; MIMIC: Medical Information Mart for Intensive Care}

\section{Acknowledgements}

Not applicable.

\section{Authors' contributions}

LW and XW discussed the content of the letter; XW wrote the manuscript. Both authors read and approved the final manuscript.

\section{Funding}

Not applicable. 
Availability of data and materials

Not applicable.

Ethics approval and consent to participate

Not applicable.

\section{Consent for publication}

Not applicable.

\section{Competing interests}

The authors declare that they have no competing interests.

\section{Author details}

'Department of Neurology, Xi'an Ninth Hospital Affiliated to Medical College of Xi'an Jiaotong University, Xi'an 710054, Shaanxi, China. ${ }^{2}$ Key Laboratory of Biomedical Information Engineering of Education Ministry, School of Life Science and Technology, Xi'an Jiaotong University, Xi'an 710049, Shaanxi, China.

Received: 7 August 2019 Accepted: 13 September 2019

Published online: 05 November 2019

\section{References}

1. Barnett MJ, Kaboli PJ, Sirio CA, Rosenthal GE. Day of the week of intensive care admission and patient outcomes - a multisite regional evaluation. Med Care. 2002:40(6):530-9.

2. Ensminger SA, Morales IJ, Peters SG, Keegan MT, Finkielman JD, Lymp JF, Afessa B. The hospital mortality of patients admitted to the ICU on weekends. Chest. 2004;126(4):1292-8.

3. Faust L, Feldman K, Chawla NV. Examining the weekend effect across ICU performance metrics. Crit Care. 2019;23(1):207.

4. Johnson AEW, Pollard TJ, Shen L, Lehman LWH, Feng ML, Ghassemi M, Moody B, Szolovits P, Celi LA, Mark RG. MIMIC-III, a freely accessible critical care database. Scientific Data. 2016;3:160035.

\section{Publisher's Note}

Springer Nature remains neutral with regard to jurisdictional claims in published maps and institutional affiliations. 